\title{
Cloning and Characterization of Cbl-associated Protein Splicing Isoforms
}

\author{
Mei Zhang, AKiko Kimura, and Alan R Saltiel
}

Cbl-associated protein (CAP) is an adaptor protein that plays important roles in both signal transduction and cytoskeleton rearrangement. Alternative splicing of the gene SORBS1 results in multiple isoforms of CAP protein. We report here the cloning of 3 new CAP isoforms, CAP2, CAP3, and CAP4, from mouse adipose tissue. RT-PCR analyses reveal that the isoform mRNAs are differentially expressed. CAP2, CAP3, and CAP4 contain a coiled-coil domain. In addition, CAP4 contains a proline-rich region, part of which exists in CAP3. Coimmunoprecipitation experiments show that CAP4 forms a homodimeric complex. While these new isoforms similarly interact with $\mathrm{Cbl}$, they exhibit varied binding specificity toward vinculin. In contrast to CAP 1 and CAP2, CAP4 does not interact with vinculin, and CAP3 binds with low affinity. Immunofluorescence analysis demonstrates differential subcellular localization of Myc-tagged CAP isoforms in 3T3-L1 adipocytes. These results suggest that these new isoforms of CAP might play different signaling roles.

\section{INTRODUCTION}

Cbl-associated protein (CAP) is a widely expressed, multifunctional adaptor protein involved in intracellular signaling $(1,2)$. $\mathrm{CAP}$ belongs to the sorbin homology (SoHo) family of proteins, including vinexin and ArgBP2, which contain 1 or 2 SoHo domains in their amino terminus and 3 adjacent $\mathrm{SH} 3$ domains in their carboxyl terminus (3). Accumulating evidence suggests that the proteins in this family are involved in the organization of both signal transduction and the cytoskeleton. CAP has been shown to play a critical role in insulin signaling (1). Cbl constitutively associates with CAP, and upon insulin stimulation, both are recruited to the insulin receptor by the adapter protein APS (4). Cbl is then phosphorylated, and the Cbl-CAP complex accumulates in lipid raft subdomains in the plasma membrane through the interaction of CAP with the raft-associated protein, flotillin (3). Phosphorylated $\mathrm{Cbl}$ recruits CrkII to the lipid raft, which initiates downstream signaling (5).

The CAP gene is highly expressed in insulin-sensitive tissues and is markedly induced during adipocyte differentiation (6). CAP transcription is increased by insulin-sensitizing thiazolidinedione drugs, by means of activation of the nuclear receptor, PPAR $\gamma$, through a PPAR response element in its promoter (7). $\mathrm{CAP}$ interacts directly with $\mathrm{Cbl}$ through its C-terminal SH3 domain. Overexpression of dominant-negative CAP mutants with deletion of the $\mathrm{SH} 3$ or SoHo domains interfere with the localization of $\mathrm{Cbl}$ to lipid rafts and specifically block insulin-stimulated Glut4 translocation and glucose uptake (1). In addition to its role in insulin action in adipocyte, CAP also has been implicated in ephrin reverse signaling in neuronal cells $(8,9)$.

The SoHo family of proteins also regulates cell adhesion and cytoskeletal organization. Previous studies have shown that CAP and ArgBP2 are associated with actin stress fibers $(2,10)$, whereas $\mathrm{CAP}$, vinexin, and ArgBP2 bind to vinculin through their $2 \mathrm{~N}$ terminal SH3 domains (11-13). Vinculin is an actin-binding cytoskeletal protein, localized at cell-extracellular matrix and cellcell adhesion sites. Exogenous expression of vinexin and CAP in fibroblasts stimulates formation of actin stress fibers and focal adhesions $(2,12)$. This effect was observed with vinculin overexpression (14), suggesting that vinculin and the SoHo family proteins may work cooperatively to regulate actin cytoskeleton rearrangement.

CAP is the product of the sorbin and SH3 domain containing 1 gene (formally designated as SORBS1). The cDNA was first cloned by screening a mouse cDNA expression library with an $\mathrm{SH} 3$ binding ligand and named SH3P12 (15). It was subsequently identified in a yeast 2-hybrid screen of a 3T3-L1 adipocyte cDNA library using $\mathrm{Cbl}$ as bait (6), therefore designated as CAP for Cbl-associated protein. Previous studies on CAP suggested the existence of various isoforms or alternatively spliced variants of this gene. At least 13 isoforms have been identified as alternative splicing products of the human SORBS1 gene (16). Northern blot analyses on multiple mouse tissues identified several CAP mRNA transcripts of different sizes. An anti-CAP antibody, which recognizes the C-terminal portion of CAP, was able to detect multiple proteins in Western Blot analyses, suggesting multiple splicing products of the mouse gene as well (6). Two splicing variants have been identified by a blot overlay method on subcellular fractions of rat liver with labeled 1-afadin and denoted as ponsin for interaction with 1-afadin and vinculin (11). Since CAP is an important adaptor protein in multiple signaling pathways, these splicing variants are potentially interesting in the effort to further delineate these pathways. We report here the cloning of 3 new splicing isoforms of CAP from mouse adipose tissue and the characterization of their protein interaction domains and subcellular localization in adipocytes.

Department of Internal Medicine and Physiology, Life Sciences Institute, University of Michigan Medical Center, Ann Arbor, MI 48109. 


\section{MATERIALS AND METHODS}

\section{Antibodies}

The antibodies to hemagglutinin (HA) (F-7), Myc (9E10), c-Cbl (C-15) were purchased from Santa Cruz Inc. The FLAG (M2) monoclonal antibody was obtained from Stratagene. The antivinculin monoclonal antibody was from Upstate Biotechnology Inc. Horseradish peroxidase-linked secondary antibodies were from Pierce Chemical Co. The Alexa Fluor secondary antibody was from Molecular Probes.

\section{RT-PCR}

Total cellular RNA was purified from 3T3-L1 fibroblasts, adipocytes, and various mouse tissues using the TRIzol reagent (Invitrogen). The synthesis of the first-strand cDNA was performed using SuperScript system from Invitrogen. For the full-length cloning of CAP variants, the white adipose tissue cDNA was amplified with PCR using the Advantage2 DNA polymerase (BD Biosciences), with the primer pair: 5'-ATCGGATTCATGAGTTCT GAATGTGATGTTGGAAGC-3' (forward) and 5'-ATCGCGGCCG CTTATAGATGTAAAGGTTTTACATAG-3' (reverse). The PCR product was separated by agarose gel electrophoresis, subcloned into the TA vector (Stratagene), and the full sequences were obtained by automated DNA sequencing.

For the multitissue RT-PCR analysis of CAP variants, cDNA from fibroblasts, adipocytes, and various mouse tissues were amplified using Taq DNA polymerase (Invitrogen) with different primer sets as follows: 5'-TGGGCTCAAGCGACTTTC-3' (forward) and 5'-CAGTTCTGGTCAATCTGTCTGTAG-3' (reverse); 5'-CCGTCTGAGGTAATAGTTGTTCC-3' (forward) and 5'-GAG CACAATGGTAGGGTTGACG-3' (reverse).

\section{Expression Constructs}

Triple-HA-tagged or Myc-tagged CAPs were constructed by subcloning CAP cDNAs in frame in the BamHI site of $\mathrm{pKH} 3$ or pKMyc vectors. Flag-tagged CAP constructs were made by subcloning CAP cDNAs in frame in the BamHI site of pcDNA-F2 vector. Flag-CAP1 $\Delta \mathrm{SH} 3$ was made as described previously (6).

\section{Cell Culture and Transfection}

COS-1 cells were grown in Dulbecco's Modified Eagle medium (DMEM) (Gibco) containing 10\% fetal bovine serum. 3T3-L1 fibroblasts were maintained in DMEM supplemented with 10\% calf serum, $100 \mathrm{U}$ of penicillin G sodium per milliliter, and $100 \mu \mathrm{g}$ of streptomycin sulfate per milliliter. Differentiation to adipocytes was induced as previously described (17). The cells then were cultured in DMEM containing 10\% fetal bovine serum. 3T3-L1 adipocytes were transfected by electroporation as described previously $(1,18)$. COS-1 cells in 60-mm-dia dishes were transfected by using FuGene 6 reagent (Roche Diagnostics).

\section{Immunoprecipitation and Immunoblotting}

Cells in 60-mm-dia dishes were washed twice with ice-cold phosphate-buffered saline and were lysed for $30 \mathrm{~min}$ at $4{ }^{\circ} \mathrm{C}$ with buffer containing $50 \mathrm{mM}$ Tris $\mathrm{HCl}$ (pH 8.0), $135 \mathrm{mM} \mathrm{NaCl}, 1 \%$ Triton X-100, 1.0 mM EDTA, 1.0 mM sodium pyrophosphate, $1.0 \mathrm{mM}$ sodium orthovanadate, $10 \mathrm{mM} \mathrm{NaF}$, and protease inhibitors
(1 tablet per $10 \mathrm{~mL}$ buffer) (Roche Diagnostics). The clarified lysates were incubated with the indicated antibodies for $1 \mathrm{~h}$ at $4{ }^{\circ} \mathrm{C}$. The immune complexes were precipitated with protein $\mathrm{A} / \mathrm{G}$ agarose (Santa Cruz Inc) for $1 \mathrm{~h}$ at $4{ }^{\circ} \mathrm{C}$ and were washed extensively with lysis buffer before solubilization in sample buffer. For antiFLAG immunoprecipitation, FLAG antibody conjugated on agarose beads (Sigma) was incubated with lysates for $2 \mathrm{~h}$ at $4{ }^{\circ} \mathrm{C}$. Bound proteins were resolved by SDS-PAGE and transferred to nitrocellulose membranes. Individual proteins were detected with the specific antibodies and visualized by blotting with horseradish peroxidase-conjugated secondary antibodies.

\section{Fluorescence Microscopy}

Electroporated 3T3-L1 adipocytes were grown on glass cover slips in 6-well dishes. Cells were fixed with 10\% formalin for $10 \mathrm{~min}$, permeabilized with $0.5 \%$ Triton X-100 for $5 \mathrm{~min}$, and blocked with $3 \%$ bovine serum albumin and $1 \%$ ovalbumin for $1 \mathrm{~h}$. Primary and Alexa Fluor secondary antibodies were used at $2 \mu \mathrm{g} / \mu \mathrm{L}$ in blocking solution, and samples were mounted on glass slides with Vectashield (Vector Laboratories). Cells were imaged using confocal fluorescence microscopy. Images were then imported into Adobe Photoshop (Adobe Systems Inc) for processing.

\section{RESULTS}

\section{Cloning of CAP Splicing Isoforms}

Total RNA was extracted from mouse white adipose tissue (WAT), and RT-PCR was performed to amplify the full-length CAP variants, using a primer set flanking the coding region of the originally cloned CAP, which is designated CAP1. Four major products were cloned and sequenced (Figure 1A). The shortest one was identical to CAP1, with the exception of 2 nucleotide substitutions, which resulted in amino acid changes of $\mathrm{T}^{206} \mathrm{~A}$ and $\mathrm{P}^{309} \mathrm{~L}$. This might represent a polymorphism between mouse tissue and the 3T3-L1 adipocytes from which CAP1 was originally cloned. The other 3 were named CAP2, CAP3, and CAP4. Analysis in GenBank indicated these 3 cDNAs were novel, although there is 1 cDNA sequence (AF521593) similar to the CAP2 cDNA (with 3 nucleotides different). These isoforms appear to be alternatively spliced products of the same gene, SORBS1, with identical amino acid compositions except for the insertion regions.

To further delineate the genomic organization of the mouse SORBS1 gene, CAP1 through CAP4 cDNAs, together with several other cDNAs obtained from GenBank that appear to be products of this same gene, were used to blast against the NCBI mouse genomic sequence databank. The mouse SORBS1 gene has been mapped to chromosome $1936.5 \mathrm{cM}$. Based on the search and alignment, the coding region of this gene is encoded by 26 known exons spanning a region of $110 \mathrm{~kb}$ (see Figure 1B). CAP1 through CAP4, ponsin1 and ponsin2, and another GenBank sequence (BC012703) are likely to be generated by alternative splicing of 6 exons (A to F) and 2 additional donor sites in exon 6abc. Comparison to the human SORBS1 gene suggests the potential existence of up to 7 more alternatively spliced exons (16).

The amino acid sequences of CAP1 through CAP4 are shown in Figure 2. Sequence analysis revealed distinct domain structures in the newly cloned isoforms. All 4 isoforms contain $2 \mathrm{~N}$-terminal 
A

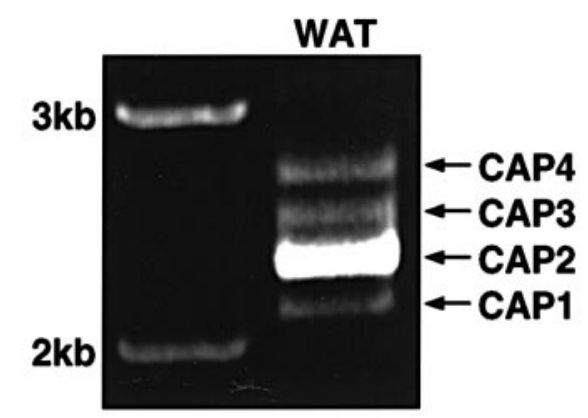

B



Isoform Name

CAP1

CAP2

CAP3

CAP4

ponsin1

ponsin2

\author{
GenBank \\ Accession number
}

U58883

AF521593 (similar)

AF078667

AF078666

BC012703

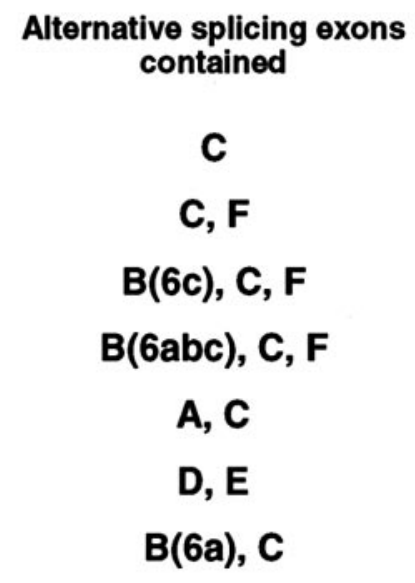

contained
colicing 
CAP1 1 MSSECDVGSSKAVVNGLASGNHGPDKDMDPTKICTGKGTVTLRASSSYRGTPSSSPVSPQESPKHESKSD

CAP2 1 MSSECDVGSSKAVVNGLASGNHGPDKDMDPTKICTGKGTVTLRASSSYRGTPSSSPVSPQESPKHESKSD

CAP3 1 MSSECDVGSSKAVVNGLASGNHGPDKDMDPTKICTGKGTVTLRASSSYRGTPSSSPVSPQESPKHESKSD

CAP4 1 MSSECDVGSSKAVVNGLASGNHGPDKDMDPTKICTGKGTVTLRASSSYRGTPSSSPVSPQESPKHESKSD

CAP1 71 EWKLSSSADTNGNAQPSPLAAKGYRSVHPSLSADKPQ

CAP2 71 EWKLSSSADTNGNAQPSPLAAKGYRSVHPSLSADKPQ

CAP3 71 EWKLSSSADTNGNAQPSPLAAKGYRSVHPSLSADKPQ

CAP4 71 EWKLSSSADTNGNAQPSPLAAKGYRSVHPSLSADKPQGATSSSSAPSEVIVVPLYLVNTDRGQGQEGTAR

CAP1

CAP2

108

CAP3

CAP4

CAP1

1A1 TPASLGPLGCVHTVPATTPAASPLTFPTLDDFIPPHLQRRPHHSQPASACGSLSPASQTSPPSPPPPLVP

GSPLLNEVSSSHIETDSQDFPPTSRPSSAYPSTTIVNPTIVLLQHNREQ

CAP2 108 -

CAP3 140 PVPEDLHRGLEPDLPGAVSSTESPLLNEVSSSHIETDSQDFPPTSRPSSAYPSTTIVNPTIVLLQHNREQ

CAP4 211 PVPEDLHRGLEPDLPGAVSSTESPLLNEVSSSHIETDSQDFPPTSRPSSAYP STTIVNPTIVLLQHNREQ

CAP1 157 QKRLSSLSDPASERRAGEQDPVPTPAELTSPGRASERRAKDASRRVVRSAQDLSDVSTDEVGIDLTIFER CAP2 157 QKRLSSLSDPASERRAGEQDPVPTPAELTSPGRASERRAKDASRRVVRSTQDLSDVSTDEVGI PLRATIER CAP3 210 QKRLSSLSDPASERRAGEQDPVPTPAELTSPGRASERRAKDASRRVVRSTQDLSDVSTDEVGI PLRATKER CAP4 281 QKRLSSLSDPASERRAGEQDPVPTPAELTSPGRASERRAKDASRRVVRSTQDLSDVSTDEVGIPLFATIEF

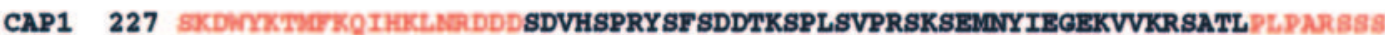

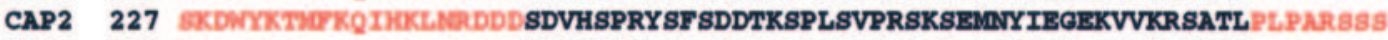

CAP3 280 BKDDMKTIOKKO IHKCLARDDD SDVHSPRYSF SDDTKSPLSVPRSKSEMAYYIEGEKVVKRSATL PLPNRBSS

CAP4 351 BKDWYKTYTKQTKKLAFDDD SDVHSPRYSFSDDTKSPLSVPRSKSEMNYIEGEKVVKRSATLPLPARSSS

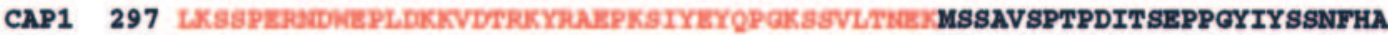

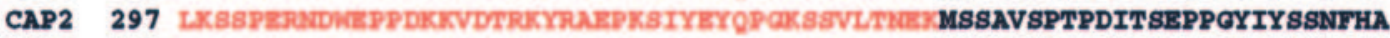

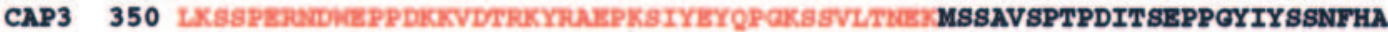

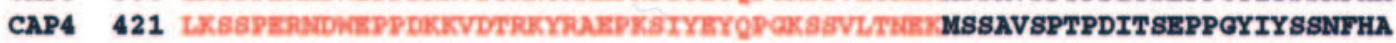

CAP1 367 VKRESDGTPGGLASLENERQIYKSVLEGGDIPLQGLSGLKRPSSSASTKDSESPRHFIPADYLESTEEFI

CAP2 367 VKRESDGTPGGLASLENERQIYKSVLEGODIPLQGLSGLKRPSSSASTKDSESPRHFIPADYLESTEEFI

CAP3 420 VKRESDGTPGGLASLENERQIYKSVLEGODIPLQGLSGLKRPSSSASTKDSESPRHFIPADYLESTEEYI

CAP4 491 VKRESDGTPGGLASLENERQIYKSVLEGGDIPLQGLSGLKRPSSSASTKDSESPRHFIPADYLESTEE / I

CAP1 437 RRRHDDK--

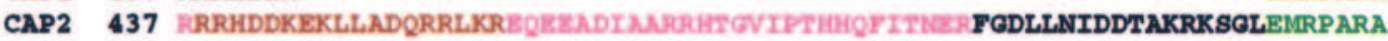

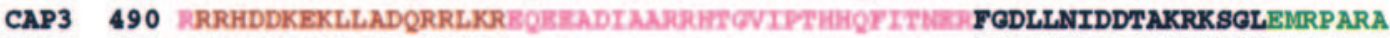

CAP4 561 RRRHDDKEKLLADQRRLKREQEEADIAMRUTTOVIPTHHOYTTIER FGDLLNIDDTAKRKSGLEMRPARA

CAP1 451 KFDFKAQTLKELPLQKGDVVYIYRQIDQNWYEGEHHGRVGIFPRTYIELLPPAEKAQPRKLAPVQVLEYG

CAP2 507 KFDFKAQTLKELPLQKGDVVYIYRQIDQNWYEGEHHGRVGIFPRTYIELLPPAEKAQPRKLAPVQVLEYG

CAP3 560 KFDFKAQTLKELPLQKGDVVYIYRQIDQNWYEGEHHGRVGIFPRTYIELLPPAEKAQPRKLAPVQVLEYG

CAP4 631 KFDFKAQTLKELPLQKGDVVYIYRQIDQNWYEGEHHGRVGIFPRTYIELLPPAEKAQPRKLAPVQVLEYG

CAP1 521 EAIAKFNFNGDTQVEMSFRKGERITLLRQVDENWYEGRIPGTSRQGIFPITYVDVLKRPLVKTPVDYIDL

CAP2 577 EAIAKFNFNGDTQVEMSFRKGERITLLRQVDENWYEGRIPGTSRQGIFPITYVDVLKRPLVKTPVDYIDL

CAP3 630 EAIAKFNFNGDTQVEMSFRKGERITLLRQVDENWYEGRIPGTSRQGIFPITYVDVLKRPLVKTPVDYIDL

CAP4 701 EAIAKFNFNGDTQVEMSFRKGERITLLRQVDENWYEGRIPGTSRQGIFPITYVDVLKRPLVKTPVDYIDL

CAP1 591 PYSSSPSRSATVSPQQPQAQQRRVTPDRSQPSLDLCSYQALYSYVPQNDDELELRDGDIVDVMEKCDDGW

CAP2 647 PYSSSPSRSATVSPQQPQAQQRRVTPDRSQPSLDLCSYQALYSYVPQNDDELELRDGDIVDVMEKCDDGW

CAP3 700 PYSSSPSRSATVSPQQPQAQQRRVTPDRSQPSLDLCSYQALYSYVPQNDDELELRDGDIVDVIIEKCDDGW

CAP4 771 PYSSSPSRSATVSPQQPQAQQRRVTPDRSQPSLDLCSYQALYSYVPQNDDELELRDGDIVDVIIEKCDDGW

CAP1 661 FVGTSRRTRQFGTFPGNYVKLYL

CAP2 717 FVGTSRRTRQFGTFPGNYVKPLYL

CAP3 770 FVGTSRRTRQFGTFPGNYVKPLYL

CAP4 841 FVGTSRRTRQFGTFPGYVKPLYL

Figure 2. Amino acid sequences of CAP isoforms and domain composition. The deduced amino acid sequences of CAP1 through CAP4 were aligned by ClustalW and Boxshade. The proline-rich region is indicated in blue; the 2 SoHo domains are in red; the coiled-coil domain is in pink; the bipartite nuclear localization signal is in brown; and the $3 \mathrm{SH} 3$ domains are in green. 


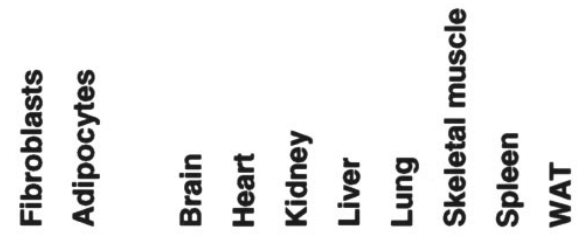

A

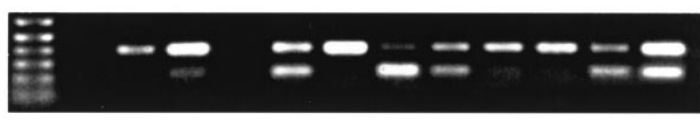

B

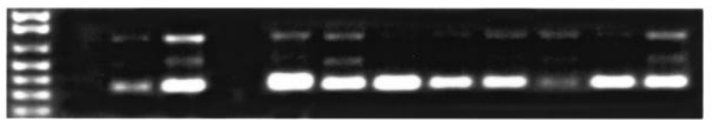

C

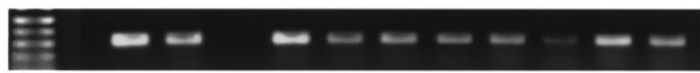

Figure 3. Tissue distribution of the new CAP isoform mRNAs. A: RT-PCR analysis using primers flanking the coiled-coil domain. Top band, isoforms contain the coiled-coil domain; bottom band, isoforms do not have the coiled-coil domain. B: RT-PCR analysis using primers flanking the prolinerich region (PRR). Top band, isoform(s) contain the PRR (CAP4); middle band, isoform(s) contain a partial PRR (CAP3); bottom band, isoforms do not contain the PRR. C: RT-PCR amplification of Racl as control.

CAP4, CAP3, and CAP4 were found in most of the tissues examined, with relatively high levels in heart and WAT, and were also induced in differentiated 3T3-L1 adipocytes (see Figure 3B). These data suggest that the CAP isoforms are expressed in a tissuespecific manner with potential distinct physiological functions. The fact that all CAP isoforms are significantly induced in 3T3-L1 adipocytes compared with fibroblasts indicates a restricted signaling role in these cells.

\section{Dimerization between CAP Isoforms}

To study the role of the newly discovered domains in mediating protein interactions, we first examined the interaction among CAP isoforms. To test the hypothesis that CAP can form homoand heterodimers through the interaction between 2 coiled-coiled domains or between the proline-rich region and the $\mathrm{SH} 3$ domains, Myc-tagged and HA-tagged CAPs were cotransfected into 3T3-L1 adipocytes by electroporation, and coimmunoprecipitation was performed to evaluate dimerization between any 2 isoforms. As shown in Figure 4A, CAP4 forms a homodimeric complex, and it also forms heterodimers with CAP1 at a much lower affinity. CAP1 does not show self-interaction, nor does it interact with CAP2. Similar experiments were done to explore the other possibilities of dimerization. The only strong interaction detected was the self-interaction of CAP4 (see Figure 4B). These data imply that intermolecular interactions between coiled-coil domains, the SH3 and proline-rich regions, or both are not sufficient to induced dimerization. Furthermore, the homodimerization of CAP4 might result from a conformational change caused by the formation of an intramolecular SH3-proline complex. This in turn, results in the exposure of the coiled-coil domain in 2 molecules that can subsequently interact with each other. It seems that the short proline-rich sequence in CAP3 is not enough to induce such a structural change.

\section{CAP Interaction with $\mathrm{Cbl}$ and Vinculin}

The presence of protein interaction domains, as well as the dimerization of the new CAP isoforms, suggests the possibility of altered ligand binding specificity of CAP proteins. CAP1 has been shown to bind to $\mathrm{Cbl}$ through its $\mathrm{C}$-terminal $\mathrm{SH} 3$ domain. This interaction is critical to the $\mathrm{CAP} / \mathrm{Cbl}$ pathway in insulin-stimulated glucose transport, since the mutant with $\mathrm{SH} 3$ domains deleted (CAP1 $\triangle \mathrm{SH} 3$ ) does not bind to $\mathrm{Cbl}$ and behaves as an dominant negative inhibitor $(1,6)$. To see whether the CAP isoforms interact with $\mathrm{Cbl}$ differently, FLAG-tagged CAPs were transfected into COS cells. Immunoprecipitation was performed with an antiFLAG antibody, and followed by anti-Cbl immunoblotting. As shown in Figure 5, endogenous $\mathrm{Cbl}$ was coprecipitated with all

\section{A}
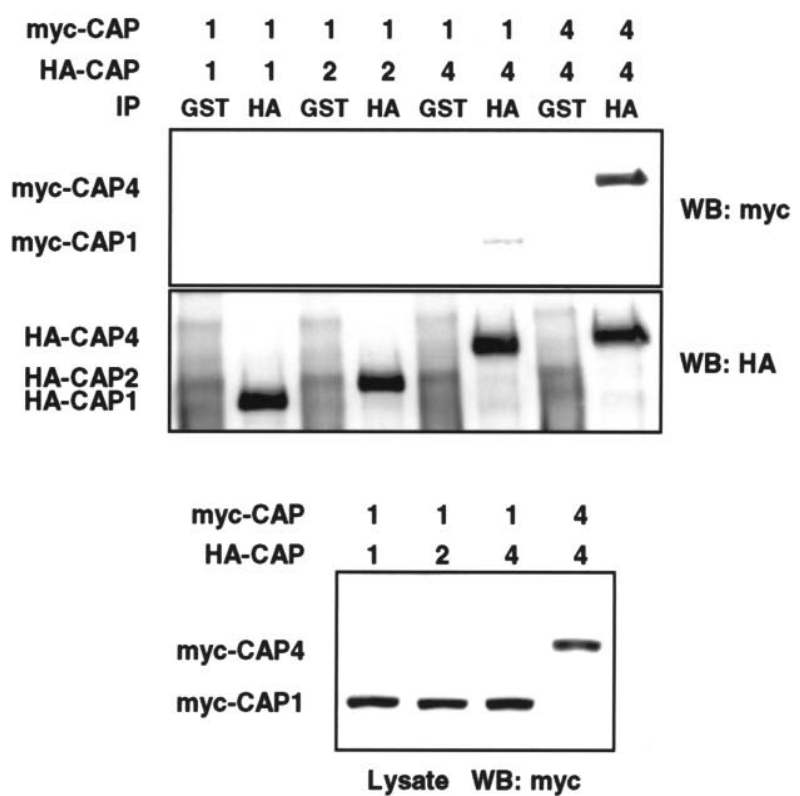

B



Lysate

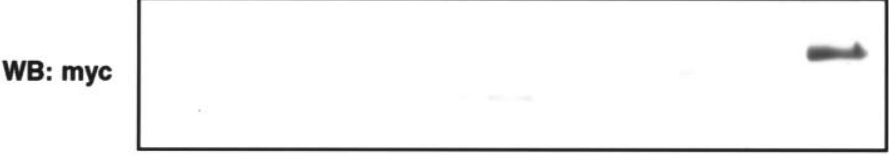

IP: HA



IP: HA

Figure 4. CAP4 forms a homodimeric complex. Myc-tagged CAP isoforms were cotransfected into 3T3-L1 adipocytes with HA-CAP isoforms (A and $B)$ or $\mathrm{HA}$ vector (B). Cell lysates were immunoprecipitated with anti-HA antibody (A and B) or anti-glutathione S-transferase antibody as control (A) and blotted with anti-Myc or anti-HA antibody. Immunoblots also were performed on whole cell lysates with anti-Myc antibody. 


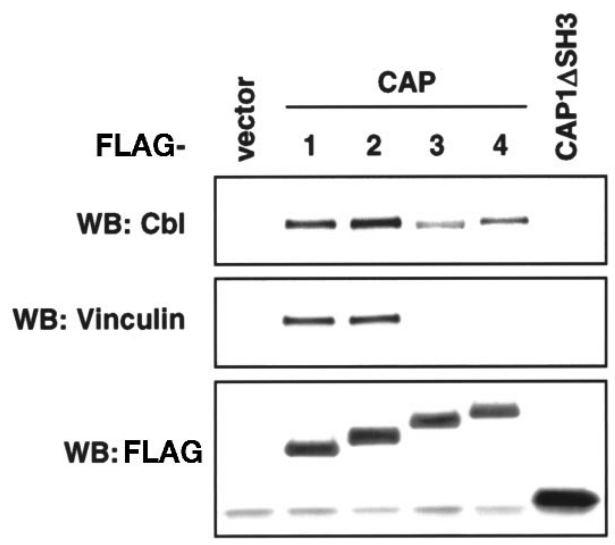

IP: anti-FLAG

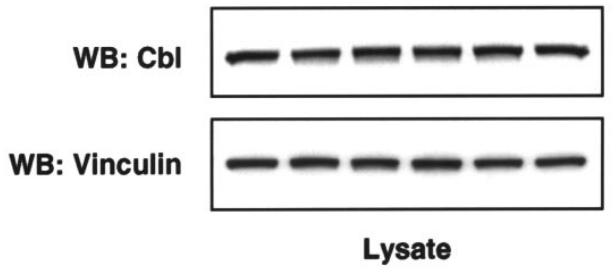

Figure 5. Interaction of CAP isoforms with $\mathrm{Cbl}$ and vinculin. Flag-tagged CAPs were transfected into COS cells and immunoprecipitated with antiFLAG antibody. The coprecipitated endogenous $\mathrm{Cbl}$ or vinculin was detected with anti-Cbl or antivinculin antibody. The total $\mathrm{Cbl}$ or vinculin in whole cell lysates is shown at the bottom.

4 isoforms, and CAP3 seems to interact with a modified form of $\mathrm{Cbl}$ with a slightly lower molecular weight. As expected, $\mathrm{CAP} 1 \Delta \mathrm{SH} 3$ did not bind to $\mathrm{Cbl}$.

CAP has been suggested to play a role in cytoskeleton rearrangement, especially the formation of focal adhesions, which may involve its binding with vinculin. This interaction is mediated through its $2 \mathrm{~N}$-terminal $\mathrm{SH} 3$ domains $(2,11)$. We examined the ability of CAP2, CAP3, and CAP4 to bind to vinculin in the same coimmunoprecipitation experiment, using an antivinculin antibody. Endogenous vinculin was coprecipitated with CAP1 and CAP2, but only bound with low affinity to CAP3 and not at all with CAP4 (see Figure 5). Taken together, these data indicate that the SH3 domains of CAP3 and CAP4 have altered proteinbinding properties, possibly through conformational changes caused by the additional proline-rich region in these isoforms.

\section{Subcellular Localization of CAPs in 3T3-L1 Adipocytes}

Since CAP1 plays a critical role in the spatial compartmentalization of insulin signaling, we decided to examine the subcellular localization of all 4 isoforms in 3T3-L1 adipocytes. Myc-tagged CAP isoforms were overexpressed in fully differentiated 3T3-L1 adipocytes by electroporation, followed by immunofluorescence staining and microscopy. As shown in Figure 6, and consistent with previous studies (4), CAP1 was localized predominantly at the plasma membrane, with some found in the cytoplasm. Interestingly, CAP2 showed nuclear localization in more than half of the cells transfected, whereas CAP4 showed patchy membrane and cytoplasmic localization with vesicular staining of unidentified structures. The staining of CAP3 was less homogenous; membrane, nuclear and/or patchy and vesicular cytoplasm staining were all observed. These results suggest that CAP isoforms might localize at different cellular compartments, which may serve differential functions in signaling.

\section{DISCUSSION}

CAP is an adaptor protein that plays important roles in several signaling pathways, including insulin-stimulated glucose transport. Alternative splicing of the same gene, SORBS1, results in multiple isoforms of CAP protein. We have cloned 3 new splicing variants of mouse CAP, CAP2, CAP3, and CAP4. All 3 isoforms contain a coiled-coil domain that is not present in CAP1. CAP3 and CAP4 have an additional proline-rich region with different length in these 2 isoforms. Coiled-coil domains are composed of amphipathic $\alpha$-helices that exhibit a heptad repeat pattern and align to form a hydrophobic interface between the supercoiled strands. Coiled-coils are found in many proteins and are often involved in mediating protein dimerization (19). A proline-rich region may represent $\mathrm{SH} 3$ or WW domain-binding motifs, which are often found in signaling proteins (21). The residues surrounding the PxxP core contribute to the optimal ligand preference, and thus help to determine the specificity of protein-protein interaction (22). The presence of these additional domains in the CAP isoforms implies novel or altered interactions.

CAP4 was found to form homodimers. The fact that CAP2 shows no self-interaction indicates that the coiled-coil domain alone is not sufficient. The interactions between CAP4 and other CAPs, all of which contain the $\mathrm{SH} 3$ domains, are weak, indicating that the potential proline-rich region in CAP4 also is not sufficient for dimerization. Moreover, no dimer formation was detected between CAP4 and CAP2, demonstrating that dimerization cannot be induced through intermolecular interactions between these isoforms. Thus, it is likely that the proline-rich region in CAP4 interacts with the SH3 domain(s) within the same molecule, which induces a conformational change of the protein, thus exposing the coiled-coil domain to form dimers. Another possibility is that CAP4 interacts with another protein that brings 2 molecules of CAP4 together. CAP3 contains the same PxxP cores as CAP4, but misses a segment of the sequence before the core region. It does not form a dimer with any other CAP isoforms, indicating that the region surrounding the PxxP cores is important in dimerization.

Previous studies showed that the 2 splicing isoforms of CAP, ponsin 1 and ponsin2, bind to and colocalize with vinculin. This interaction is mediated through the binding of the first $2 \mathrm{SH} 3$ domains of ponsin to the proline-rich hinge region of vinculin. We examined the interaction between vinculin and the newly cloned CAP isoforms. CAP2 binds to vinculin in a manner similar to CAP1, whereas CAP3 showed a low-level interaction. No interaction was detected between CAP4 and vinculin. Interestingly, CAP3 also exhibits a lower affinity for $\mathrm{Cbl}$, although CAP4 binds in a manner similar to what is observed for CAP1 and CAP2. These data suggest that the additional proline-rich sequences in CAP3 and CAP4 may differentially restrict the availability of the first $2 \mathrm{SH} 3$ domains, through induction of protein conformation changes and/or interactions with other proteins, thus modulating vinculin or $\mathrm{Cbl}$ binding to CAP. 
myc-CAP1

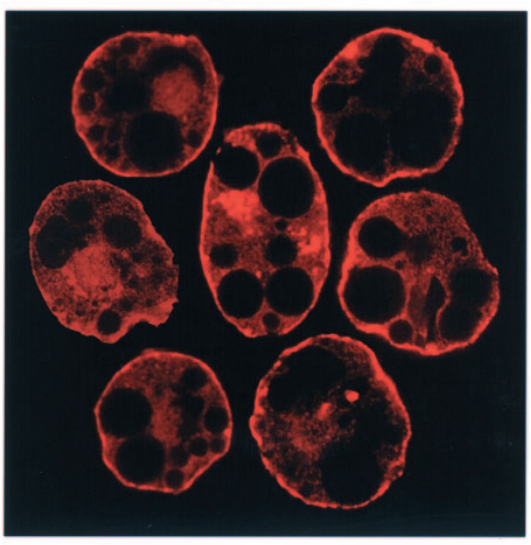

myc-CAP3

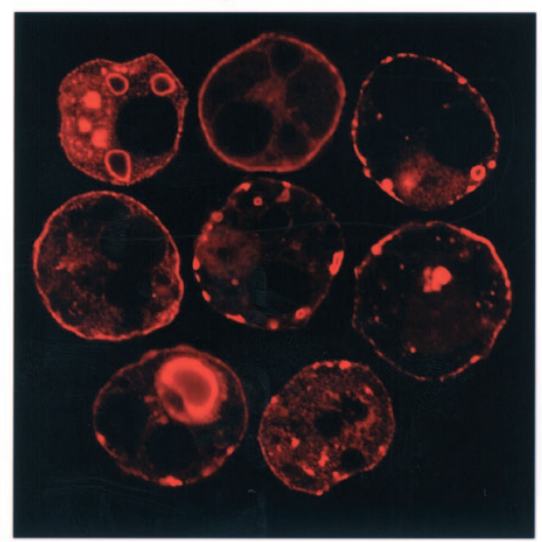

myc-CAP2

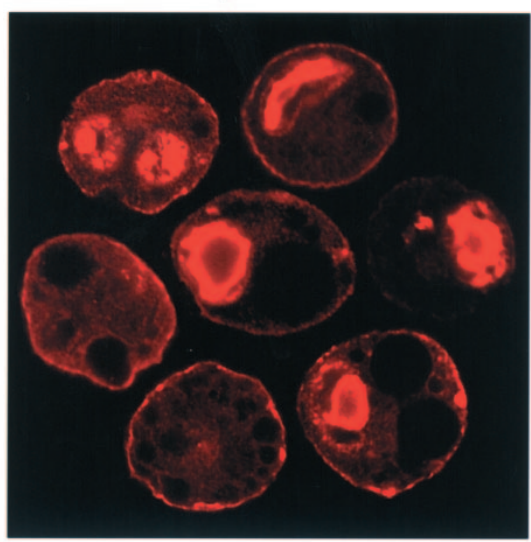

myc-CAP4



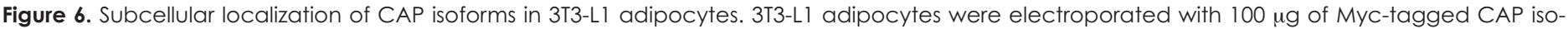
forms. Cells were plated on cover slips and allowed to recover for $30 \mathrm{~h}$. Myc-CAPs were visualized by indirect immunofluorescent staining.

The subcellular localization of adaptor proteins is important in the compartmentalization of signaling pathways, through the recruitment of proteins to specified subcellular domains. CAP1 plays such a role in insulin-stimulated glucose transport by bringing tyrosine phosphorylated $\mathrm{Cbl}$ to the lipid raft domain of the plasma membrane. We analyzed the localization of ectopically expressed CAP isoforms in 3T3-L1 adipocytes. While the plasma membrane localization of CAP2, CAP3, and CAP4 was similar to that exhibited by CAP1 in some cells, CAP2 showed strong nuclear staining in more than half of the cells, and CAP3 also exhibited nuclear localization. This suggested that the interactions of CAPs with vinculin were not an important determinant of localization in the adipocytes, although they may direct localization in other cell types. Previous immunostaining of fibroblasts using an anti-CAP antibody, which recognizes all the isoforms, showed some localization in the nucleus $(9,11)$. Sequence analysis identified a bipartite nuclear localization signal in 1 of the alternatively spliced exon (F), which is present in CAP2, CAP3, and CAP4 (see Figure 2). The recently cloned human homologue of CAP2, R85, together with its binding partner, ataxin-7 (a protein responsible for the neurodegenerative disease spinocerebellar ataxia 7), is localized in the nucleus in transfected COS cells and the neuronal intranuclear inclusions in brain (23). In addition, the progesterone receptor has been shown to bind to the C-terminal SH3 domain of CAP (24). Furthermore, ArgBP2 is localized in the nucleus as well as its localization at stress fibers (10). The ArgBP2 binding partner $\mathrm{c}-\mathrm{Abl}$ exhibits similar localization and has been shown to shuttle between stress fibers and the nucleus to integrate cell adhesion and cell cycle signals (25). Taken together, these data suggest potential nuclear functions of specific CAP isoforms.

The altered ligand binding specificity and differential subcellular localization of CAP isoforms suggest the possibility that these isoforms might carry out distinct functions. The varied expression pattern of each isoform in different tissues implies tissue-specificity of its functions. The significant induction of expression of all the isoforms in fully differentiated adipocytes indicates the overall importance of the CAP proteins in these cells. Whether these isoforms indeed serve distinct signaling functions needs further investigation. Moreover, the differences in domain structures and localization of CAP isoforms also imply potential novel ligand, which may be identified in future studies.

\section{ACKNOWLEDGMENTS}

This work was supported by NIH Grant DK60591. We thank Drs Jun Liu and Shian-Huey Chiang for technical assistance and helpful discussions. 
Address correspondence and reprint requests to Alan $R$ Saltiel, Department of Internal Medicine and Physiology, Life Sciences Institute, University of Michigan Medical Center, Ann Arbor, MI 48109-0650. Phone: 734-615-9787; fax: 734-936-2888; e-mail: saltiel@ umich.edu.

Submitted January 3, 2003; accepted for publication January 17, 2003.

\section{REFERENCES}

1. Baumann CA et al. (2000) CAP defines a second signalling pathway required for insulin-stimulated glucose transport. Nature 407:202-7.

2. Ribon V, Herrera R, Kay BK, Saltiel AR (1998) A role for CAP, a novel, multifunctional Src homology 3 domain-containing protein in formation of actin stress fibers and focal adhesions. J. Biol. Chem. 273:4073-80.

3. Kimura A, Baumann CA, Chiang SH, Saltiel AR (2001) The sorbin homology domain: a motif for the targeting of proteins to lipid rafts. Proc. Natl. Acad. Sci. U.S.A. 98:9098-103

4. Liu J, Kimura A, Baumann CA, Saltiel AR. (2002) APS facilitates C-Cbl tyrosine phosphorylation and GLUT4 translocation in response to insulin in 3T3-L1 adipocytes. Mol. Cell Biol. 22:3599-609.

5. Chiang SH et al. (2001) Insulin-stimulated GLUT4 translocation requires the CAPdependent activation of TC10. Nature 410:944-8.

6. Ribon V, Printen JA, Hoffman NG, Kay BK, Saltiel AR. (1998) A novel, multifuntional C-Cbl binding protein in insulin receptor signaling in 3T3-L1 adipocytes. Mol. Cell Biol. 18:872-9.

7. Baumann CA, Chokshi N, Saltiel AR, Ribon V. (2000) Cloning and characterization of a functional peroxisome proliferator activator receptor- $\gamma$-responsive element in the promoter of the CAP gene. J. Biol. Chem. 275:9131-5.

8. Cowan CA, Henkemeyer M. (2002) Ephrins in reverse, park and drive. Trends Cell. Biol. 12:339-46.

9. Cowan CA, Henkemeyer M. (2001) The SH2/SH3 adaptor Grb4 transduces B-ephrin reverse signals. Nature 413:174-9.

10. Wang B, Golemis EA, Kruh GD. (1997) ArgBP2, a multiple Src homology 3 domain-containing, Arg/Abl- interacting protein, is phosphorylated in v-Abl- transformed cells and localized in stress fibers and cardiocyte Z-disks. J. Biol. Chem. 272:17542-50.

11. Mandai $\mathrm{K}$ et al. (1999) Ponsin/SH3P12: an I-afadin- and vinculin-binding protein localized at cell-cell and cell-matrix adherens junctions. J. Cell Biol. 144:1001-17.

12. Kioka $\mathrm{N}$ et al. (1999) Vinexin: a novel vinculin-binding protein with multiple SH3 domains enhances actin cytoskeletal organization. J. Cell Biol. 144:59-69.

13. Kawabe $\mathrm{H}$ et al. (1999) nArgBP2, a novel neural member of ponsin/ArgBP2/vinexin family that interacts with synapse-associated protein 90/postsynaptic density-95-associated protein (SAPAP). J. Biol. Chem. 274:30914-8.

14. Geiger B et al. (1992) Cytoplasmic control of cell adhesion. Cold Spring Harb. Symp. Quant. Biol. 57:631-42.

15. Sparks AB, Hoffman NG, McConnell SJ, Fowlkes DM, Kay BK. (1996) Cloning o ligand targets: systematic isolation of $\mathrm{SH} 3$ domain-containing proteins. Nat Biotechnol. 14:741-4.

16. Lin WH et al. (2001) Cloning, mapping, and characterization of the human sorbin and $\mathrm{SH} 3$ domain containing 1 (SORBS1) gene: a protein associated with c-Abl during insulin signaling in the hepatoma cell line Hep3B. Genomics 74:1220.

17. Rubin CS, Lai E, Rosen OM. (1977) Acquisition of increased hormone sensitivity during in vitro adipocyte development. J. Biol. Chem. 252:3554-7.

18. Min J et al. (1999) Synip: a novel insulin-regulated syntaxin 4-binding protein mediating GLUT4 translocation in adipocytes. Mol. Cell 3:751-60.

19. Lupas A, Van Dyke M, Stock J. (1991) Predicting coiled coils from protein sequences (http://www.ch.embnet.org/software/COILS_form.html) Science 252:1162-4.

20. Lupas A. (1996) Coiled coils: new structures and new functions. Trends Biochem. Sci. 21:375-82

21. Macias MJ, Wiesner S, Sudol M. (2002) WW and SH3 domains, two different scaffolds to recognize proline-rich ligands. FEBS Lett. 513:30-7.

22. Kay BK, Williamson MP, Sudol M. (2000) The importance of being proline: the interaction of proline-rich motifs in signaling proteins with their cognate domains. FASEB. J. 14:231-41.

23. Lebre AS et al. (2001) Ataxin-7 interacts with a Cbl-associated protein that it recruits into neuronal intranuclear inclusions. Hum. Mol. Genet. 10:1201-13.

24. Boonyaratanakornkit $V$ et al. (2001) Progesterone receptor contains a prolinerich motif that directly interacts with $\mathrm{SH} 3$ domains and activates $\mathrm{C}$-Src family tyrosine kinases. Mol. Cell. 8:269-80.

25. Lewis JM, Baskaran R, Taagepera S, Schwartz MA, Wang JY. (1996) Integrin regulation of C-Abl tyrosine kinase activity and cytoplasmic- nuclear transport. Proc. Natl. Acad. Sci. U.S.A. 93:15174-9. 